\title{
Higher thyrotropin leads to unfavorable lipid profile and somewhat higher cardiovascular disease risk: evidence from multi-cohort Mendelian randomization and metabolomic profiling
}

Nicolien A. van Vliet ${ }^{1}$, Maxime M. Bos ${ }^{1,2}$, Carisha S. Thesing ${ }^{3}$, Layal Chaker ${ }^{2,4,5}$, Maik Pietzner ${ }^{6}$, Evelyn Houtman Matt J. Neville ${ }^{8,9}$, Ruifang Li-Gao ${ }^{10}$, Stella Trompet ${ }^{1}$, Rima Mustafa ${ }^{11}$, Fariba Ahmadizar ${ }^{2}$, Marian Beekman", Mariska Bot ${ }^{3}$, Kathrin Budde ${ }^{12}$, Constantinos Christodoulides ${ }^{8,9}$, Abbas Dehghan ${ }^{11,13}$, Christian Delles ${ }^{14}$, Paul Elliott ${ }^{11,13,15,16}$, Marina Evangelou ${ }^{17}$, He Gao ${ }^{11}$, Mohsen Ghanbari ${ }^{2}$, Antonius E. van Herwaarden ${ }^{18}$, M. Arfan Ikram², Martin Jaeger ${ }^{19,20}$, J. Wouter Jukema ${ }^{21,22}$, Ibrahim Karaman ${ }^{11,13}$, Fredrik Karpe ${ }^{8,9}$, Margreet Kloppenburg ${ }^{10,23}$, Jennifer M. T. A. Meessen ${ }^{7,24}$, Ingrid Meulenbelt ${ }^{7}$, Yuri Milaneschi ${ }^{3}$, Simon P. Mooijaart ${ }^{1,25}$, Dennis O. Mook-Kanamori ${ }^{10,26}$, Mihai G. Netea ${ }^{19}$, Romana T. Netea-Maier ${ }^{19}$, Robin P. Peeters ${ }^{4,5}$, Brenda W. J. H. Penninx ${ }^{3}$, Naveed Sattar ${ }^{27}$, P. Eline Slagboom,28, H. Eka D. Suchiman ${ }^{7}$, Henry Völzke ${ }^{29}$, Ko Willems van Dijk ${ }^{30,31,32}$, Raymond Noordam ${ }^{1 *}$ (D), Diana van Heemst ${ }^{1 *}$ and BBMRI Metabolomics Consortium $^{7}$

\begin{abstract}
Background: Observational studies suggest interconnections between thyroid status, metabolism, and risk of coronary artery disease (CAD), but causality remains to be proven. The present study aimed to investigate the potential causal relationship between thyroid status and cardiovascular disease and to characterize the metabolomic profile associated with thyroid status.

Methods: Multi-cohort two-sample Mendelian randomization (MR) was performed utilizing genome-wide significant variants as instruments for standardized thyrotropin (TSH) and free thyroxine (fT4) within the reference range. Associations between TSH and fT4 and metabolic profile were investigated in a two-stage manner: associations between TSH and fT4 and the full panel of 161 metabolomic markers were first assessed hypothesisfree, then directional consistency was assessed through Mendelian randomization, another metabolic profile platform, and in individuals with biochemically defined thyroid dysfunction.
\end{abstract}

\footnotetext{
* Correspondence: r.noordam@lumc.nl; d.van_heemst@lumc.nl

${ }^{1}$ Department of Internal Medicine, Section of Gerontology and Geriatrics,

Leiden University Medical Center, PO Box 9600, 2300 RC Leiden, The

Netherlands

Full list of author information is available at the end of the article
}

(c) The Author(s). 2021 Open Access This article is licensed under a Creative Commons Attribution 4.0 International License, which permits use, sharing, adaptation, distribution and reproduction in any medium or format, as long as you give appropriate credit to the original author(s) and the source, provide a link to the Creative Commons licence, and indicate if changes were made. The images or other third party material in this article are included in the article's Creative Commons licence, unless indicated otherwise in a credit line to the material. If material is not included in the article's Creative Commons licence and your intended use is not permitted by statutory regulation or exceeds the permitted use, you will need to obtain permission directly from the copyright holder. To view a copy of this licence, visit http://creativecommons.org/licenses/by/4.0/. The Creative Commons Public Domain Dedication waiver (http://creativecommons.org/publicdomain/zero/1.0/) applies to the data made available in this article, unless otherwise stated in a credit line to the data. 


\begin{abstract}
Results: Circulating TSH was associated with 52/161 metabolomic markers, and fT4 levels were associated with 21/ 161 metabolomic markers among 9432 euthyroid individuals (median age varied from 23.0 to 75.4 years, 54.5\% women). Positive associations between circulating TSH levels and concentrations of very low-density lipoprotein subclasses and components, triglycerides, and triglyceride content of lipoproteins were directionally consistent across the multivariable regression, MR, metabolomic platforms, and for individuals with hypo- and hyperthyroidism. Associations with fT4 levels inversely reflected those observed with TSH. Among 91,810 CAD cases and 656,091 controls of European ancestry, per 1-SD increase of genetically determined TSH concentration risk of CAD increased slightly, but not significantly, with an OR of 1.03 (95\% Cl 0.99-1.07; $p$ value 0.16 ), whereas higher genetically determined fT4 levels were not associated with CAD risk (OR 1.00 per SD increase of fT4; $95 \% \mathrm{Cl}$ 0.96-1.04; $p$ value 0.59).
\end{abstract}

Conclusions: Lower thyroid status leads to an unfavorable lipid profile and a somewhat increased cardiovascular disease risk.

Keywords: Thyroid hormones, Coronary artery disease, Metabolomics, Mendelian randomization

\section{Background}

Hypothyroidism, defined by high thyroid stimulating hormone (TSH) and low free thyroxine (fT4) levels, and subclinical hypothyroidism, defined by high TSH and fT4 within the reference range, are associated with higher total cholesterol, low-density lipoprotein cholesterol (LDL-c), and triglyceride levels [1, 2], and subclinical hypothyroidism has been associated with higher coronary artery disease (CAD) risk [3]. However, two recent randomized placebo-controlled trials on levothyroxine treatment in older adults with subclinical hypothyroidism did not find a reduction in cardiovascular events $[4,5]$, possibly due to a lack of statistical power [6].

Mendelian randomization (MR) studies [7] and studies using metabolomics data can further elaborate on the possible causal role of thyroid status in CAD [8]. Previous MR studies on thyroid status and CAD were performed in multi-ancestry populations [9-12], while thyroid function [13], prevalence of thyroid dysfunction $[13,14]$, and risk of myocardial infarction [15] all vary by ancestry. Moreover, genetic variants for thyroid parameters were discovered in European-ancestry populations only [16]. We hypothesized that performing MR in an exclusively European sample could provide a more accurate effect estimation. In addition, metabolomic profiling can be used as intermediate phenotype, to investigate early subclinical stages of diseases, especially when considering the lipoprotein subclasses and their contents $[17,18]$. Recently, findings from a Brazilian cohort showed already promising results showing subclinical thyroid function to be related to unfavorable lipid profile using a metabolomics platform $[19,20]$.

We aimed to investigate the potential causal role of thyroid status in cardiovascular disease by assessment of the association between TSH and fT4 levels and CAD using MR in European-ancestry cohorts. Additionally, we investigated the association between thyroid status and metabolomic profile in two stages. First, associations between TSH and fT4 concentrations within the reference range were tested for the complete panel of 161 metabolomic markers. Next, robustness of associations between TSH and fT4 and the metabolomic markers identified in stage one, was tested with MR and a different NMR-metabolomics platform. Since the multivariable-adjusted regression and $M R$ analyses methods are sensitive to different sources of bias, residual confounding and unbalanced horizontal pleiotropy respectively, triangulation of evidence can contribute to causal inference of observational findings [21]. The consistency of associations with metabolomic markers was also examined in individuals with thyroid dysfunction.

\section{Methods}

Study populations for multivariable-adjusted regression analyses on the metabolomic profile

We strived to include as much cohorts as possible with data on exposure and outcome being measured in European-ancestry participants. In the end, data from six European-ancestry cohorts were used for first stage analysis of circulating metabolomic marker concentrations and thyroid status; the 500 Functional Genomics Study (500FG) $(n=421)$ [22], the Genetics, Arthrosis and Progression study (GARP) $(n=321)$ [23], the Leiden Longevity Study (LLS) $(n=486)$ [24], the Netherlands Study of Depression and Anxiety (NESDA) $(n=2906)$ [25], PROSPER $(n=5316)$ [26], and the Rotterdam Study (RS) $(n=1690)$ [27] (detailed description in Additional File 1: Supplementary Materials). We used data from Study of Health in Pomerania (SHIP) as validation $(n=983)$ using different metabolomic profiling methods [28]. Each participating study obtained written informed 
consent from all participants and approval from the appropriate local institutional review boards.

\section{Thyroid parameters for multivariable regression analyses}

For the multivariable regression analyses, TSH and fT4 were measured according to a standardized protocol (See Additional File 1: supplementary Materials). For analyses on TSH and fT4 within the reference range, cohort-specific reference ranges were used after which TSH and fT4 levels were inverse normal transformed to approximate normal distribution and facilitate comparison between cohorts. Biochemical thyroid dysfunction was also based on cohort-specific reference ranges; overt hyperthyroidism was defined by TSH levels below the reference range and fT4 levels above the reference range, overt hypothyroidism was defined by either TSH $>20$ $\mathrm{mIU} / \mathrm{L}$ or TSH below $20 \mathrm{mIU} / \mathrm{L}$ but above the reference range and fT4 below the reference range.

\section{Genetic instruments for TSH and fT4}

Genetic instruments for TSH and fT4 concentrations were extracted from the largest genome-wide association studies (GWAS) meta-analysis on thyroid function comprising 72,167 European-ancestry participants [16]. A total of 62 independent single nucleotide polymorphisms (SNPs) were identified for circulating TSH (GWASbased $9.4 \%$ explained variance) and 31 independent SNPs for circulating fT4 (GWAS-based 4.8\% explained variance) [16] (Additional File 1: Online Table 1). Median F-statistics was 54 (range 32 to 576 ) for the TSH instruments and 43 (range 30 to 394) for the fT4 instruments.

\section{Outcome sources for metabolomic profile}

Data for MR analyses on thyroid status and metabolomic profile were derived from four sources; MAGNETIC consortium $(n=24,925$; downloaded from: http://www.computationalmedicine.fi/data\#NMR GWAS) [29], the Oxford Biobank $(n=6616)$ [30], the Netherlands Epidemiology of Obesity Study $(n=$ 4734) [31], and PROspective Study of Pravastatin in the Elderly at Risk (PROSPER) $(n=2343)$ [26] (Additional File 1: Supplementary Materials). Data of the MAGNETIC consortium was publicly available. For the other studies, linear regression analyses were performed between the SNPs and standardized metabolomic marker concentrations (mean $=0, \mathrm{SD}=1$ ), adjusted for age, sex, and up to ten principal components. Findings were validated in the Airwave Health Monitoring Study (Airwave) $(n=2021)$ that used a different NMR platform [32].
Metabolomic profile measurements for multivariableadjusted regression and Mendelian randomization analyses

We used metabolomic profile measurements performed on a high-throughput proton NMR platform (Nightingale Health Ltd., Helsinki, Finland) [33]. This method provides quantification of lipoprotein subclass profiling with lipid concentrations within 14 subclasses, fatty acid composition and other small molecules including glycolysis-related metabolites, amino acids, and ketone bodies [33] (total 161 metabolomic markers). Out of the 161 metabolic markers, 116 were included in the GWAS of the MAGNETIC consortium. Metabolomic profiling for SHIP and Airwave was generated by Bruker IVDr LIpoprotein Subclass Analysis (B.I.-LISA; Bruker Biospin, Rheinstetten, Germany) [34-36]. Out of the 105 quantified lipoprotein subclasses, 57 subclasses overlapped with Nightingale. Methodological details are described in the Additional File 1: Supplementary Materials.

\section{Outcome sources for CAD}

For MR analyses on thyroid status and CAD, we used data from three studies with European-ancestry participants; CARDIoGRAM consortium (22,233 cases and 64,762 controls; downloaded from: http://www. cardiogramplusc4d.org/data-downloads/) [37], UK Biobank (52,946 cases and 393,549 controls) [38], and FinnGen (16,631 cases and 197,780 controls; freeze 5; downloaded from: https://www.finngen.fi/en/access_ results) [39] to perform MR analyses using maximum sample size and to examine consistency of the MR results across the different cohorts. Case definitions are described in the Additional File 1: Supplementary Materials.

\section{Statistical analyses}

For analyses on circulating metabolomic marker concentrations, values were natural log-transformed and subsequently standardized for analyses.

For the multivariable regression analyses, a prespecified analysis plan and syntax were distributed among cohorts. Population characteristics were derived as number (percentage) for categorical variables, mean and SD for normally distributed variables and median, and interquartile range (IQR) for non-normally distributed variables. Multivariable linear regression analyses were performed locally, and summarized results were collected centrally for quality control and meta-analysis. The main analysis was adjusted for age, sex, body mass index (BMI), and smoking (current versus former or never), which were considered major confounders. Given potential heterogeneity among cohorts included in the multivariable regression analyses, the meta-analysis 
comprised a random-effects model according to restricted maximum likelihood (REML). Sensitivity analyses were done with participants excluded who used thyroid therapy or lipid-lowering medication (defined by ATC codes $\mathrm{H} 03$ and $\mathrm{C} 10$, respectively) or medication for treatment of diabetes mellitus (DM), reported a history of thyroid disease or DM, or had fasting glucose > $7 \mathrm{mmol} / \mathrm{L}$. Results for the multivariable regression analyses are presented as the association between one SD higher TSH and fT4 levels within the reference range and metabolomic marker concentrations in SD with 95\% CI. For associations of thyroid dysfunction, regressed differences in circulating metabolomic marker concentrations in SD are shown for overt hyper- and hypothyroid individuals compared to euthyroid individuals. Analyses in this stage were hypothesis-free, and therefore, Bonferroni correction was applied based on 37 uncorrelated metabolomic markers (as previously applied [18]), resulting in a two-sided $p$ value threshold of less than $1.34 \times$ $10^{-3}(0.05 / 37)$. Circos plots were used to summarize and visually compare the $\mathrm{fT} 4$ and $\mathrm{TSH}$ results. Circos plots were created using EpiViz (version 0.1.0, https://github. com/mattlee821/EpiViz/), a Shiny web application and R package built using $\mathrm{R}$ (version 3.6.2) and Shiny (version 1.4.0). EpiViz adapts and builds on the Circlize [40] and ComplexHeatmap [41] R packages to create Circos plots compatible with association analysis data.

\section{Mendelian randomization analyses}

Two-sample MR analyses were conducted using summary-level data from relatively independent GWAS from exposure and outcomes [42]. We extracted the association of each genetic variant for TSH and fT4 from summary data of GWAS for circulating metabolomic markers and CAD. All palindromic SNPs, which are SNPs with an effect allele frequency close to 0.5 in combination with alleles that correspond to nucleotides that pair with each other, were excluded prior to analyses, as being default in the TwoSampleMR package. Furthermore, to prevent overestimation of the precision of the causal effects, we excluded all SNPs in linkage disequilibrium at $R^{2}>0.001$ from analyses as well. From each of the remaining SNPs, we calculated the explained variance (as $(\beta \times \sqrt{ }(2 \times$ minor allele frequency $\times(1-$ minor allele frequency)) $\left.)^{2} \times 100\right)$ and F-statistics (as $(\beta /$ standard error) ${ }^{2}$ ).

Our main analyses were inverse variance-weighted (IVW) analyses, which provide a weighted mean estimate of the association of the genetically determined exposure and the outcome assuming none of the instruments were invalid using additive random effects [43]. We performed weighted median estimator (WME), MR Egger regression, and MR pleiotropy residual sum and outlier (MR-PRESSO) analyses as sensitivity analyses to take into account possible bias caused by directional pleiotropy [43, 44]. MR-Egger is similar to IVW but does not force the regression line (i.e., of the SNP-thyroid status trait association on the SNP-metabolomic measure association) through the intercept. MR-Egger is statistically less efficient (providing wider confidence intervals) but provides a causal estimate (i.e., the regression slope) that is corrected for directional horizontal pleiotropy and a non-zero intercept which is an indication of the existence of directional pleiotropy. The weighted-median estimator is valid if more than $50 \%$ of the weight of the genetic instrument is from valid variants (i.e., if one single SNP or several SNPs jointly contributing $50 \%$ or more of the weight in the MR analysis exhibit horizontal pleiotropy the calculated effect estimate may be biased). We first performed MR analyses on each dataset separately and subsequently meta-analyzed the summary estimates using fixed-effects models. Effect estimates for MR analyses with metabolomic profile represent the mean difference in metabolomic marker concentration in SD per 1-SD increase in TSH and fT4 levels with 95\% CI. For MR analyses on CAD, results are presented as odds ratio (OR) per 1-SD genetically determined increase in circulating TSH and fT4 levels with 95\% confidence interval (CI). As all MR analyses were hypothesisdriven, a conventional two-sided $p$ value of less than 0.05 was considered statistically significant.

All analyses and data visualization were performed in $R$ version 3.6.1 [45] supplemented with the following packages; MRCIEU/TwoSampleMR [46], rondolab/MRPRESSO [44], metafor [47], ggplot2 [48], and ggforestplot [49].

\section{Results}

Associations between TSH and fT4 within the reference range and metabolomic profile

Participant characteristics of the stage 1 cohorts

For the multivariable regression analyses, 11,140 adults from six cohorts were included. A total of 9432 (84.7\%) were euthyroid, 194 (1.7\%) had hypothyroidism, 721 (6.5\%) had subclinical hypothyroidism, 263 (2.4\%) had subclinical hyperthyroidism, and 54 (0.5\%) had hyperthyroidism (Additional File 1: Online Table 2). Among euthyroid individuals, the median age varied from 23.0 to 75.4 years and $54.5 \%$ of these participants were women (Table 1). Median TSH levels ranged between 1.73 and $2.13 \mathrm{mIU} / \mathrm{L}$, mean fT4 levels ranged between 15.6 to $16.4 \mathrm{pmol} / \mathrm{L}$, and thyroid medication was used by 185 individuals $(2.0 \%)$ and lipid-modifying medication by 2694 individuals $(28.6 \%)$.

\section{Stage 1 analyses}

TSH levels were associated with 52/161 metabolomic marker concentrations and fT4 levels associated with 
Table 1 Population characteristics of biochemically euthyroid individuals in included cohorts $(n=9432)$

\begin{tabular}{|c|c|c|c|c|c|c|}
\hline & $\begin{array}{l}500 \text { FG } \\
N=362\end{array}$ & $\begin{array}{l}\text { GARP } \\
N=230\end{array}$ & $\begin{array}{l}\text { LLS } \\
N=419\end{array}$ & $\begin{array}{l}\text { NESDA } \\
N=2467\end{array}$ & $\begin{array}{l}\text { PROSPER } \\
N=4513\end{array}$ & $\begin{array}{l}\text { RS } \\
N=1441\end{array}$ \\
\hline Age in years (median (IQR)) & $23.0(21.0-26.0)$ & $\begin{array}{l}59.8(55.1- \\
65.5)\end{array}$ & $65.7(61.8-70.4)$ & $43.0(30.0-53.0)$ & $75.4(72.9-78.3)$ & $\begin{array}{l}68.9(65.2- \\
73.3)\end{array}$ \\
\hline Women & $200(55.2)$ & $181(78.7)$ & $202(48.2)$ & $1594(64.6)$ & 2195 (48.6) & $773(53.6)$ \\
\hline Current smoker & $47(13.1)^{\mathrm{a}}$ & $38(16.5)$ & $51(12.2)^{c}$ & $984(39.9)$ & $1231(27.3)^{9}$ & $204(14.2)^{\mathrm{h}}$ \\
\hline BMI (median (IQR)) & $\begin{array}{l}22.3(20.8- \\
24.2)^{\mathrm{b}}\end{array}$ & $\begin{array}{l}26.0(24.0- \\
29.0)\end{array}$ & $\begin{array}{l}26.3(24.2- \\
28.6)^{\mathrm{d}}\end{array}$ & $\begin{array}{l}24.6(22.0- \\
28.0)^{f}\end{array}$ & $\begin{array}{l}26.2(23.8- \\
28.9)^{9}\end{array}$ & $\begin{array}{l}26.4(24.2- \\
29.0)\end{array}$ \\
\hline TSH (median (IQR)) & $2.09(1.59-2.79)$ & $\begin{array}{l}1.76(1.27- \\
2.34)\end{array}$ & $2.13(1.54-2.89)$ & $2.07(1.47-2.80)$ & $1.73(1.22-2.44)$ & $\begin{array}{l}1.76(1.27- \\
2.51)\end{array}$ \\
\hline fT4 (mean (SD)) & $16.4(2.1)$ & $15.8(1.8)$ & $15.6(1.9)$ & $15.6(2.0)$ & $15.6(1.9)$ & $15.7(1.8)$ \\
\hline History of diabetes mellitus & $0(0.0)$ & $3(1.3)$ & $20(6.0)^{\mathrm{e}}$ & $103(4.2)$ & $471(10.4)$ & $149(10.4)^{\mathrm{i}}$ \\
\hline Lipid-lowering medication use & $0(0.0)$ & $8(3.5)$ & $55(16.6)^{\mathrm{e}}$ & $184(7.5)$ & $2248(49.8)$ & $199(14.7)^{\mathrm{j}}$ \\
\hline History of thyroid disease & $0(0.0)$ & N.A. & N.A. & $62(2.5)$ & N.A. & $111(7.7)$ \\
\hline Thyroid medication use & $0(0.0)$ & $3(1.3)$ & $7(2.1)^{\mathrm{e}}$ & $34(1.4)$ & $113(2.5)$ & $28(1.9)$ \\
\hline $\begin{array}{l}\text { Medication use influencing the thyroid } \\
\text { gland }\end{array}$ & $0(0.0)$ & N.A. & $1(0.3)^{\mathrm{e}}$ & $3(0.1)$ & $12(0.3)$ & N.A. \\
\hline
\end{tabular}

Results are shown as $n$ (\%) unless indicated otherwise. Abbreviations: 500 FG 500 Functional Genomics Study, GARP the Genetics, Arthrosis and Progression study, LLS the Leiden Longevity Study, NESDA the Netherlands Study of Depression and Anxiety, PROSPER PROspective Study of Pravastatin in the Elderly at Risk, RS the Rotterdam Study, BMI body mass index, TSH thyroid stimulating hormone, fT4 free thyroxin, N.A. not available

anformation on 360 individuals

bInformation on 356 individuals

Information on 410 individuals

Information on 407 individuals

e Information on 331 individuals

f Information on 2465 individuals

ginformation on 4511 individuals

${ }^{\mathrm{h}}$ Information on 1435 individuals

information on 1438 individuals

'Information on 1352 individuals

21/161 metabolomic markers (Fig. 1; Additional File 1: Online Table 3). Higher TSH levels were predominantly associated with higher concentrations of very lowdensity lipoprotein (VLDL) subclasses and components, higher triglyceride concentrations, and higher triglyceride content of lipoproteins. Associations with fT4 were largely an inverse reflection of those observed with TSH. Fluid balance parameters (creatinine and albumin) appeared specific for TSH, while ketone bodies appeared specific for fT4.

\section{Stage 2 analyses}

For the metabolomic markers associated with TSH in the first stage, second-stage analyses with $\mathrm{MR}$ and/or Bruker platform were performed to assess directional consistency of the results (Fig. 2; Additional File 1: Online Table 4). For the MR meta-analysis of TSH and metabolomic markers, 41/52 metabolomic markers identified in the first stage were available. For the majority of these (34/41), associations observed with MR and multivariable regression were directionally consistent. These markers included various subclasses of VLDL cholesterol, fatty acids, and triglyceride subclasses. Inconsistent associations between $\mathrm{MR}$ and multivariable regression comprised associations of TSH with triglyceride content of IDL- and small HDL-cholesterol particles, albumin, various amino acids, glycolysis related metabolites, and inflammatory markers. Overlapping coverage between Nightingale and Bruker was found for 23/52 of the metabolomic markers identified in the first stage that all showed comparable associations in multivariable regression analyses across both platforms.

For the metabolomic markers associated with fT4 in the first stage, second-stage analyses were performed to assess robustness (Additional File 1: Online Figure 1; Additional File 1: Online Table 4). Of the only 4/21 metabolomic markers from the first stage present in available genetics data, the association with acetoacetate was directionally consistent in MR, but the observations with the amino acids and triglyceride content of IDL-cholesterol were not. A total of 9/21 markers were present on both the Nightingale and Bruker platforms (e.g., VLDL cholesterol subclass, HDL and LDL triglyceride content); all showed directional consistency with similar effect estimates.

Restricting the study sample to those without thyroid or lipid-lowering medication use or metabolic disease, produced similar results as observed in our main analyses (Additional File 1: Online Table 5). Sensitivity analyses for MR 


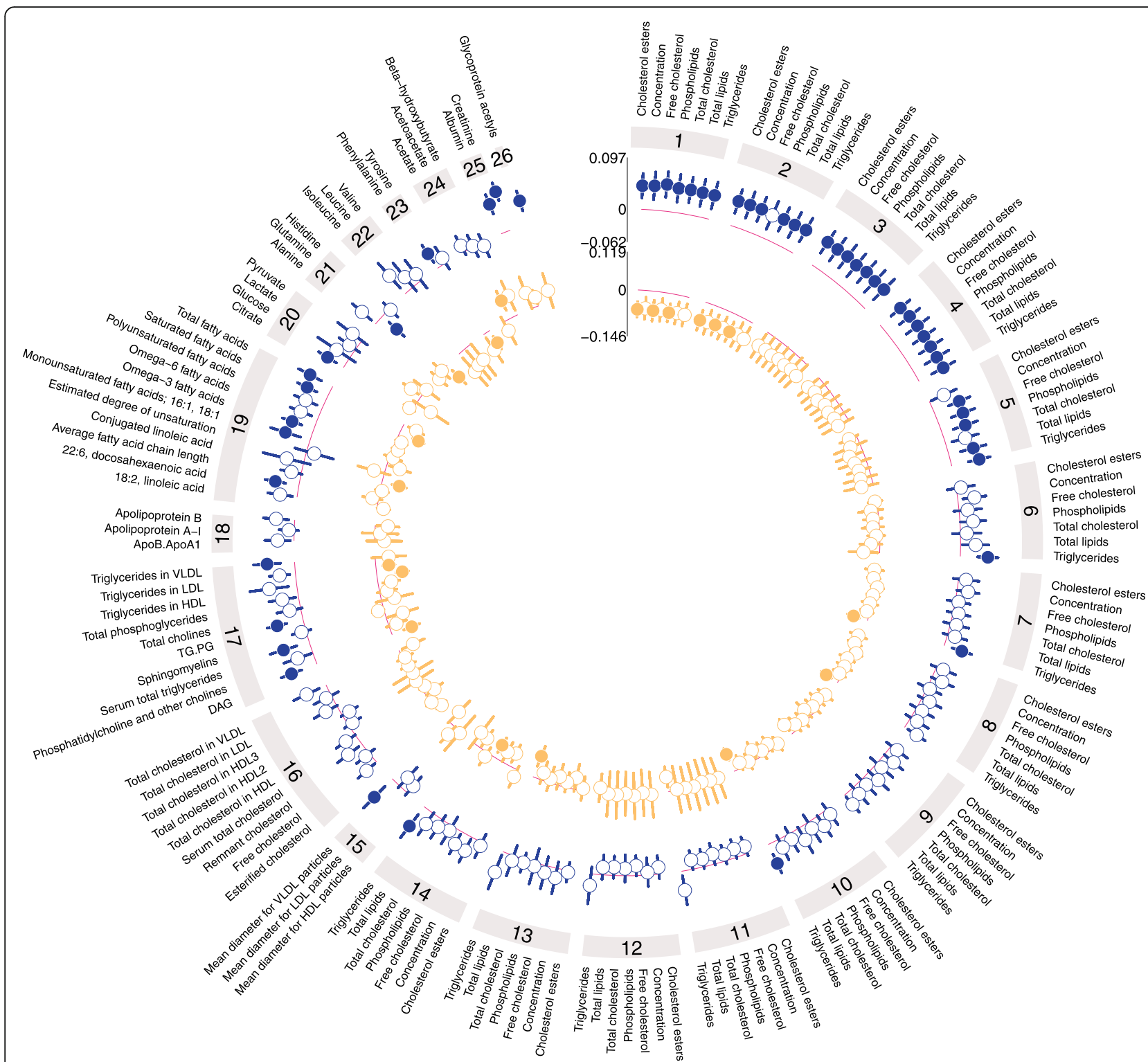

Fig. 1 First stage associations between standardized TSH and fT4 within the reference range and 161 Nightingale platform metabolomic markers ( $N=$ 9353). Point estimates represent the standardized change in metabolomic marker concentration per standard deviation change in TSH, adjusted for age, sex, body mass index, and smoking. Red bars indicate positive associations; blue bars indicate negative associations. Hollow effect estimates were not statistically significant after correction for multiple testing $\left(p\right.$ value $\left.<1.34 \times 10^{-3}\right)$. (1) Extreme large VLDL. (2) Very large VLDL. (3) Large VLDL. (4) Medium VLDL. (5) Small VLDL. (6) Very small VLDL. (7) IDL. (8) Large LDL. (9) Medium LDL. (10) Small LDL. (11) Very large HDL. (12) Large HDL. (13) Medium HDL. (14) Small HDL. (15) Lipoprotein particle size. (16) Cholesterol. (17) Glycerides and phospholipids. (18) Apolipoproteins. (19) Fatty acids. (20) Glycolysis-related metabolites. (21) Amino acids. (22) Branched-chain amino acids. (23) Aromatic amino acids. (24) Ketone bodies. (25) Fluid balance. (26) Inflammation. HDL, high-density lipoprotein; LDL, low-density lipoprotein; VLDL, very-low density lipoprotein

and the MR findings on the Bruker platform were consistent with the main findings (Additional File 1: Online Table 6).

\section{Association between biochemical thyroid dysfunction and metabolomic markers identified in relation to TSH and}

fT4

Consistency of the observed metabolomic profile was additionally explored in individuals with hyperthyroidism $(n=54)$ and hypothyroidism $(n=194)$. Virtually all metabolomic markers identified in the first stage analyses with TSH and fT4 were directionally consistent with hypo- and hyperthyroidism (Additional File 1: Online Figure 2). For TSH, 44/52 and 5/52 and for fT4, 14/ 21 and 2/31 metabolomic markers reached nominal significant associations $(p<0.05)$ with respectively hypothyroidism and hyperthyroidism (Additional File 1: Online Table 7). Overall, the VLDL subclasses and components associated with TSH and fT4 within the 


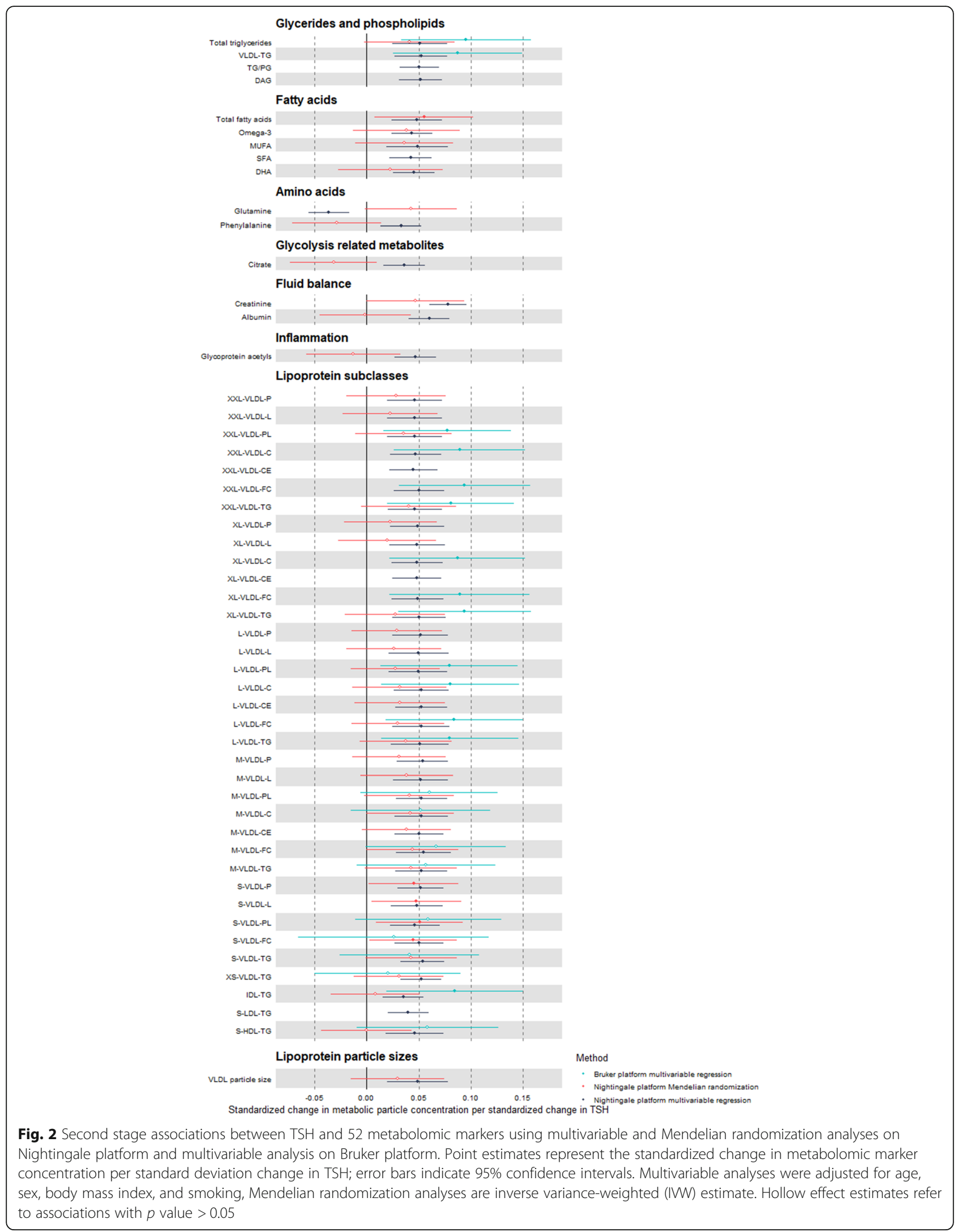


reference range appeared to associate stronger with hypothyroidism than with hyperthyroidism.

\section{Associations between genetically determined TSH and fT4} and coronary artery disease

Within the multi-cohort MR study comprising 91,810 cases with CAD and 656,091 controls, per 1-SD increase of genetically determined TSH concentration CAD risk increased with an OR of 1.03 (95\% CI 0.99-1.07; $p$ value 0.16) (Fig. 3). Genetically determined fT4 concentrations were not associated with CAD (OR 1.00 per 1-SD increase of genetically determined fT4; 95\% CI 0.96-1.04; $p$ value 0.89 ). Heterogeneity between cohorts was low; all study-level effect estimates were congruent and $I^{2}<$ 21.00\%. The MR Egger and WME were consistent with the IVW estimates (Fig. 3), although some evidence was observed in the meta-analysis that higher TSH was associated with higher CAD risk (OR 1.06 per 1-SD increase of genetically determined TSH; 95\% CI 1.001.10). The MR Egger intercepts did not deviate from zero and MR-PRESSO did not indicate distortion by outliers (Additional File 1: Online Table 8).

\section{Discussion}

Using a mixed-methods approach of multi-cohort multivariable regression analysis and MR, we identified a robust metabolomic profile associated with lower thyroid status within the reference range, comprising higher concentrations of VLDL subclasses and components, higher triglyceride concentrations, and higher triglyceride content of lipoproteins. These associations were directionally consistent in patients with thyroid disorders. In addition, in this multi-cohort MR study on thyroid status and CAD in an exclusively European-ancestry

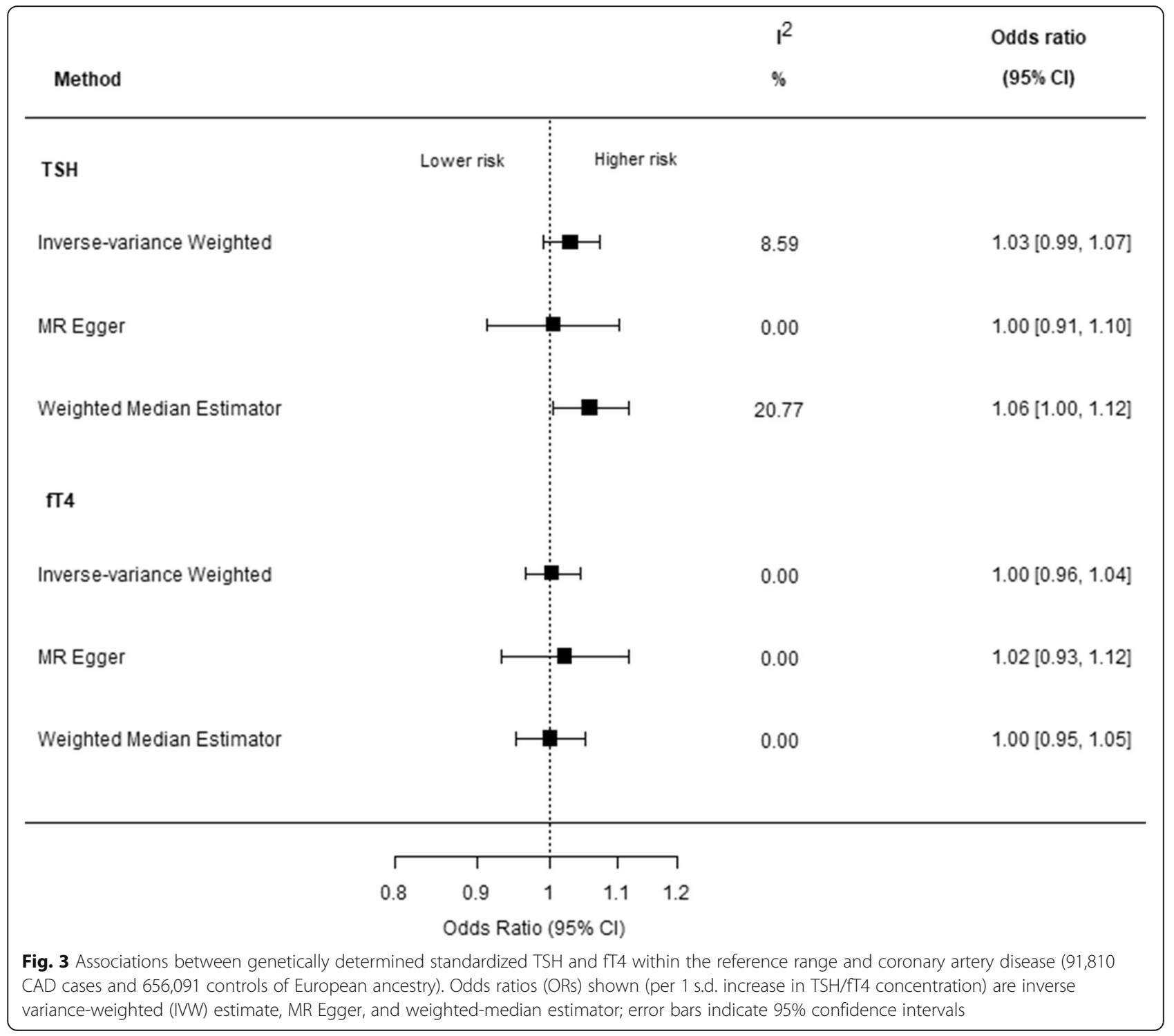


population, genetically determined higher TSH concentrations were associated with a slightly higher CAD risk (particularly in the WME analysis).

As far as we are aware, this is the first multi-cohort study investigating the association between thyroid status and metabolomic profile. Although various smaller studies were performed previously, results were difficult to compare due to the variety of platforms, techniques, and sample types (i.e., plasma, serum, urine) [50]. Nevertheless, in line with our results, most studies indicated a role of thyroid hormones in lipid metabolism. No MR studies on thyroid status parameters and detailed metabolomic profile have been published to date.

Previous multi-ancestry MR studies found no evidence for an association between thyroid status and CAD [911 . While one MR study found evidence for an association between hypothyroidism and CAD, no associations were observed between genetically determined TSH or fT4 concentrations and CAD [12]. We hypothesize that the difference between our and previous studies might be because we restricted our analyses to Europeanancestry individuals to decrease the risk of population stratification bias. Future research should assess the extent of confounding by population stratification in MR studies on thyroid status.

Multiple mechanisms may underlie the observed associations between thyroid status, CAD, and metabolomic profile. An important function of thyroid hormone is to stimulate the mobilization and breakdown of cholesterol and bile acids as well as the de-novo synthesis of fatty acids and their uptake by peripheral tissues, especially oxidative tissues such as skeletal muscle, heart, and liver [51]. Thyroid status could alter hepatic clearance of lipoproteins and reverse transport of cholesterol [52]. Consequently, disturbances in thyroid hormone availability and action may result in disturbances in the balance between lipid mobilization/synthesis on the one hand and uptake/clearance on the other hand (reviewed by Duntas et al. [53]). In case of higher TSH/lower thyroid hormone, the rate of cholesterol mobilization will be higher than the rate of its degradation, resulting in higher circulating cholesterol levels, which form a substrate for lipid peroxidation and may enhance oxidative stress as well as low grade chronic inflammation. In parallel, higher $\mathrm{TSH} /$ lower fT4 may also result in a decreased clearance of TG-rich lipoproteins, which may further aggravate the adverse lipid profile. Lower thyroid status could therefore result in accumulation of fatty acids in VLDL particles and free triglycerides in the circulation, resulting in the observed metabolomic profile. Interestingly, the metabolomic profile that we observed for lower thyroid status resembles that identified previously for myocardial infarction [54]. Therefore, a plausible pathway would be from low thyroid status via unfavorable lipid profile which could provide a substrate for oxidative stress and inflammatory processes to CAD. Although several other potential mediating factors should be considered, including endothelial dysfunction, hypertension and alterations in coagulation [55].

The stronger effect of TSH compared to fT4 on CAD risk in our study should be interpreted with caution. Though the genetic variants for fT4 were all strongly associated with higher circulating fT4 levels, some of these genes do not result in higher intracellular thyroid hormone signaling [56]. These shortcomings of the fT4 genetic risk score were also demonstrated previously in context of thyroid status and atrial fibrillation [57]. Furthermore, interpretation of the association for genetically determined higher TSH with CAD cannot be specified to either variation within the reference range or including (sub)clinical hypothyroidism, as many of the genetic variants associated with higher TSH within the reference range also associated with TSH levels above the reference range [16].

The present study has a number of strengths. Owing to the multi-cohort setting, we could compile large study populations for our analyses. Beside the statistical benefits of large sample sizes, multi-cohort studies allow for surpassing cohort-specific effects and therefore contribute to identifying robust and generalizable associations. Apart from assessing consistency of associations between study populations and the possibility of neglecting some important confounders not present in all contributing cohorts (e.g., specific drug use), we made efforts to triangulate our findings, using different epidemiological research methods, on the metabolomic profile. To assess (biological) consistency and robustness, the metabolomic markers associated with variation in TSH and fT4 within the reference range were tested in individuals with thyroid disorders and in studies using another NMR metabolomic profiling platform. The directional consistency among these different approaches indicates robust results.

Our study also has certain limitations. The MR study on the association of TSH and fT4 with CAD was performed in European-ancestry individuals only and is therefore not directly extrapolatable to other ethnicities. Furthermore, although we attempted to include as much cohorts as possible in our study, cohorts with both exposure and outcome were scarce and therefore the power of some of the analyses, in particular the validation analyses, is limited. Despite claims for causal inference in MR studies, caution is warranted for bias due to horizontal pleiotropy, selection bias and latent structure [58-60]. The study population used for first stage analyses of associations between TSH and fT4 and metabolomic profile included a considerable proportion of individuals using lipid-lowering medication (30\%) or 
with a history of DM (8\%). Nevertheless, results from our second stage and sensitivity analyses excluding participants with thyroid or lipid-lowering medication and those with a history of thyroid disease or DM were in line with our first stage results. However, not all metabolomic markers could be tested in the second stage analysis due to low overlap of markers in available data and platforms. Furthermore, both the MR study on genetically determined TSH and fT4 with CAD and with circulating metabolites suffered from some sample overlap between exposure and outcome study populations, which might cause bias, though the extent appears limited [61]. Moreover, multivariable MR to formally assess mediation of the association between TSH and CAD by metabolomic profile was not possible, as specific genetic instruments for separate metabolomic markers are currently unavailable due to the high (genetic) correlation between the different components and subclasses.

\section{Conclusions}

We found indications for potentially causal elevated risks of unfavorable lipid profile and a somewhat increased risk cardiovascular disease in individuals with TSH on the upper limits of the reference range. However, the effect sizes were small and therefore do not justify widespread treatment with levothyroxine for prevention of cardiovascular disease. Nevertheless, the present study adds novel insights in the cardiovascular risk profile of those with altered thyroid hormone levels.

\begin{abstract}
Abbreviations
500FG: 500 Functional Genomics Study; Airwave: Airwave Health Monitoring Study; ATC: Anatomical Therapeutic Chemical; BMI: Body mass index; CAD: Coronary artery disease; CARDIoGRAM: Coronary Artery Disease Genome wide Replication and Meta-analysis; Cl: Confidence interval; DM: Diabetes mellitus; fT4: Free thyroxine; GARP: Genetics, Arthrosis and Progression study; GWAS: Genome-wide association study; HDL: High-density lipoprotein; IDL: Intermediate-density lipoprotein; IQR: Interquartile range; IVW: Inverse-variance weighted; LDL: Low-density lipoprotein; LLS: Leiden Longevity Study; MR: Mendelian randomization; MR-PRESSO: Mendelian randomization pleiotropy residual sum and outlier; NEO: Netherlands Epidemiology of Obesity; NESDA: Netherlands Study of Depression and Anxiety; NMR: Nuclear magnetic resonance; OR: Odds ratio; PROSPER: Prospective Study of Pravastatin in the Elderly at Risk; REML: Restricted maximum likelihood; RS: Rotterdam Study; SD: Standard deviation; SHIP: Study of Health in Pomerania; SNP: Single nucleotide polymorphism; TSH: Thyrotropin/thyroid-stimulating hormone;

WME: Weighted median estimator; VLDL: Very low-density lipoprotein
\end{abstract}

\section{Supplementary Information}

The online version contains supplementary material available at https://doi. org/10.1186/s12916-021-02130-1.

Additional file 1: Extended Methods. Supplementary Table 1. Associations of individual genetic instruments for TSH and fT4 with CAD. Supplementary Table 2. Population characteristics of included cohorts $(n=11,140$. Supplementary Table 3. First stage associations between standardized TSH and fT4 within the reference range and 161 metabolomic markers. Supplementary Table 4. Second stage associations between metabolomic markers associated with TSH and fT4

\section{in Mendelian randomization analyses and Bruker platform.}

Supplementary Table 5. Sensitivity analyses for metabolomic markers associated with TSH and fT4 in a restricted population without thyroid medication, lipid-lowering medication or history of diabetes. Supplementary Table 6. Sensitivity analyses for Mendelian randomization analyses of metabolomic markers and TSH and fT4. Supplementary Table 7. Associations between metabolomic markers associated with TSH and fT4 and biochemical thyroid dysfunction. Supplementary Table 8. Results for sensitivity analyses for MR on thyroid status and CAD. Supplementary Figure 1. Second stage associations between fT4 and 21 metabolomic markers. Supplementary Figure 2. Association of thyroid dysfunction with metabolomic markers identified for TSH and fT4 in first stage.

\section{Acknowledgements}

We thank all study participants for their generous cooperation. We are grateful to all supporting staff and the participating general practitioners and pharmacists. Special thanks go to Drs. N. Riyazi, J. Bijsterbosch, H.M. Kroon, and I. Watt.

\section{Authors' contributions}

Conception: NAVV, MMB, RN, DvH; DESIGN OF THE WORK: NAvV, MMB, RN, DvH; acquisition, analysis: NAvV, MMB CST, LC, MP, EH, MJN, RLG, ST, RM, FA, MB1, MB2, KB, CC, AD, CD, PE, ME, HG, MG, AEvH, MAI, MJ, JWJ, IK, FK, MK, JMTAM, IM, YM, SPM, DOM-K, MGN, RTN-M, RPP, MWJHP, NS, PES, HEDS, HV, $\mathrm{KWvD}, \mathrm{RN}, \mathrm{DvH}$; interpretation of data: NAvV, RN, DvH; drafted the work or substantively revised it: NAvV, MMB, RN, DvH; approved the submitted version: NAVV, MMB CST, LC, MP, EH, MJN, RLG, ST, RM, FA, MB1, MB2, KB, CC, $A D, C D, P E, M E, H G, M G, A E v H, M A I, M J, J W J$, IK, FK, MK, JMTAM, IM, YM, SPM, DOM-K, MGN, RTN-M, RPP, MWJHP, NS, PES, HEDS, HV, KWVD, RN, DVH; agreed accountability for own contribution to the work: NAvV, MMB CST, LC, MP, EH, MJN, RLG, ST, RM, FA, MB1, MB2, KB, CC, AD, CD, PE, ME, HG, MG, AEVH, MAI, MJ, JWJ, IK, FK, MK, JMTAM, IM, YM, SPM, DOM-K, MGN, RTN-M, RPP, MWJHP, NS, PES, HEDS, HV, KWVD, RN, DvH. All authors read and approved the final manuscript.

\section{Funding}

This work was supported by the European Commission project THYRAGE (Horizon 2020 research and innovation program, 666869) and BBMRI-NL, a research infrastructure financed by the Dutch Research Council (NWO, 184.021.007 and 184.033.111).

The PROSPER study was supported by an investigator initiated grant obtained from Bristol-Myers Squibb. JWJ is an Established Clinical Investigator of the Netherlands Heart Foundation (grant 2001 D 032). Support for genotyping was provided by the seventh framework program of the European commission (grant 223004) and by the Netherlands Genomics Initiative (Netherlands Consortium for Healthy Aging grant 050-060-810).

The infrastructure for the NESDA study (www.nesda.nl) is funded through the Geestkracht program of the Netherlands Organisation for Health Research and Development (ZonMw, grant number 10-000-1002) and financial contributions by participating universities and mental health care organizations (VU University Medical Center, GGZ inGeest, Leiden University Medical Center, Leiden University, GGZ Rivierduinen, University Medical Center Groningen, University of Groningen, Lentis, GGZ Friesland, GGZ Drenthe, Rob Giel Onderzoekscentrum).

The Rotterdam Study is funded by Erasmus Medical Center and Erasmus University, Rotterdam, Netherlands Organization for the Health Research and Development (ZonMw), the Research Institute for Diseases in the Elderly (RIDE), the Ministry of Education, Culture and Science, the Ministry for Health, Welfare and Sports, the European Commission (DG XII), and the Municipality of Rotterdam.

This work in SHIP was funded by grants from the German Federal Ministry of Education and Research (BMBF, grants 01ZZ0403, 01ZZ0103, 01GI0883, AtheroSysMed 03IS2061B), the Ministry for Education, Research and Cultural Affairs, as well as the Ministry of Social Affairs of the Federal State of Mecklenburg-West Pomerania. This work is also part of the research project Greifswald Approach to Individualized Medicine (GANI_MED). The GANI_MED consortium is funded by the Federal Ministry of Education and Research and the Ministry of Cultural Affairs of the Federal State of Mecklenburg-West Pomerania (03IS2061A). 
The Leiden Longevity Study has received funding from the European Union's Seventh Framework Programme (FP7/2007-2011) under grant agreement number 259679, the Innovation-Oriented Research Program on Genomics (SenterNovem IGE05007), the Centre for Medical Systems Biology and the Netherlands Consortium for Healthy Ageing (grant 050-060-810), all in the framework of the Netherlands Genomics Initiative, Netherlands Organization for Scientific Research (NWO), and BBMRI-NL, a Research Infrastructure financed by the Dutch Research Council (NWO 184.021.007 and 184.033.111)

The 500FG was supported by an ERCConsolidator Grant (310372) and an INCONTROL CVON grant from the Dutch Heart Foundation (CVON2012-03 and CVON2018-27). MGN was supported by an ERC Advanced Grant (833247) and a Spinoza Grant of the Netherlands Organization for Scientific Research (94-212).

The GARP study is supported by Leiden University Medical Centre, Pfizer Groton, Connecticut, USA, and the Dutch Arthritis Society. Furthermore, the research leading to these results has received funding from the Biobanking and BioMolecular resources Research Infrastructure the Netherlands (BBMRINL) (complementation project CP2013-84), the Dutch Scientific Research council NWO/ZonMW VICI scheme (nr. 91816631/528) and the Dutch Arthritis Society.

The OBB and Oxford BioResource are funded by the NIHR Oxford Biomedica Research Centre (BRC). CC is supported by an Intermediate Clinical Research Fellowship to (FS/16/45/32359) from the British Heart Foundation and FK from a BHF programme grant (RG/17/1/32663).

The NEO study is supported by the participating Departments, the Division and the Board of Directors of the Leiden University Medical Centre, and by the Leiden University, Research Profile Area 'Vascular and Regenerative Medicine'.

The Airwave Health Monitoring Study was funded by the UK Home Office (780- TETRA, 2003-2018) and is currently funded by the MRC and ESRC (MR/ R023484/1) with additional support from the NIHR Imperial College Biomedical Research Centre in collaboration with Imperial College NHS Healthcare Trust. PE acknowledges support from the MRC and PHE (MR/L01341X/1, 2014-2019) and currently from the MRC for the MRC Centre for Environment and Health (MR/S019669/1). PE is supported by the UK Dementia Research Institute which receives funding from UK DRI Ltd funded by the UK Medical Research Council, Alzheimer's Society and Alzheimer's Research UK. PE is associate director of the Health Data Research UK London funded by a consortium led by the UK Medical Research Council. RM was supported by the President's PhD Scholarships from Imperial College London.

None of the funders influenced data collection, analyses or interpretation, or the decision to publish this research. The views expressed are those of the authors and not necessarily those of the sponsors.

\section{Availability of data and materials}

The data used for the analyses on CAD are all publicly available. The summary-level data for CARDloGRAM (http://www.cardiogramplusc4d.org/ data-downloads/) and FinnGen (https://finngen.gitbook.io/documentation/ data-download) are freely obtainable. The data for UK Biobank are accessible after approval (https://www.ukbiobank.ac.uk/). All other datasets used and/or analyzed during the current study are available from the corresponding authors on reasonable request.

\section{Declarations}

\section{Ethics approval and consent to participate}

The different cohorts contributing to the meta-analyses of the multivariable adjusted and MendelianRandomization studies were accepted by the medical ethical committees from the Leiden University MedicalCenter (GARP, LLS, NEO, PROSPER), Erasmus Medical Center (RS), Radboud University Medical Center (500FG),VU University Medical Center (NESDA), Oxfordshire Clinical Research Ethics Committee (OBB), University ofGreifswald (SHIP), and the National Health Service Multi-site Research Ethics Committee(AIRWAVE; MREC/ 13/NW/0588).

\section{Consent for publication}

Not applicable.

\section{Competing interests}

DOMK is a part-time research consultant at Metabolon, Inc. All others declare no conflicts of interest.

\section{Author details}

${ }^{1}$ Department of Internal Medicine, Section of Gerontology and Geriatrics, Leiden University Medical Center, PO Box 9600, 2300 RC Leiden, The Netherlands. ${ }^{2}$ Department of Epidemiology, Erasmus MC, Rotterdam, The Netherlands. ${ }^{3}$ Amsterdam UMC, Vrije Universiteit, Department of Psychiatry, Amsterdam Public Health research institute, Amsterdam, The Netherlands. ${ }^{4}$ Academic Center for Thyroid Diseases, Erasmus MC, Rotterdam, The Netherlands. ${ }^{5}$ Department of Internal Medicine, Erasmus MC, Rotterdam, The Netherlands. ${ }^{6}$ Computational Medicine, Berlin Institute of Health $(\mathrm{BIH})$, Charité-Universitätsmedizin Berlin, Berlin, Germany. ${ }^{7}$ Department of Biomedical Data Sciences, section of Molecular Epidemiology, Leiden University Medical Center, Leiden, The Netherlands. ${ }^{8} \mathrm{NIHR}$ Oxford Biomedical Research Centre, Oxford University Hospitals Foundation Trust, Oxford, UK. ${ }^{9}$ Radcliffe Department of Medicine, Oxford Centre for Diabetes, Endocrinology, and Metabolism, University of Oxford, Oxford, UK. ${ }^{10}$ Department of Clinical Epidemiology, Leiden University Medical Center, Leiden, The Netherlands. ${ }^{11}$ MRC Centre for Environment and Health, Department of Epidemiology and Biostatistics, School of Public Health, Imperial College London, London, UK. ${ }^{12}$ Institute of Clinical Chemistry and Laboratory Medicine, University Medicine Greifswald, Greifswald, Germany.

${ }^{13}$ Dementia Research Institute at Imperial College London, London, UK. ${ }^{14}$ Institute of Cardiovascular and Medical Sciences, College of Medical, Veterinary and Life Sciences, University of Glasgow, Glasgow, UK. ${ }^{15} \mathrm{NIHR}$ Biomedical Research Centre, Imperial College London, London, UK. ${ }^{16} \mathrm{BHF}$ Imperial College Centre for Research Excellence, Imperial College London, London, UK. ${ }^{17}$ Department of Mathematics, Faculty of Natural Sciences, Imperial College London, London, UK. ${ }^{18}$ Department of Laboratory Medicine, Radboud Laboratory for Diagnostics (RLD), Radboud University Medical Center, Nijmegen, The Netherlands. ${ }^{19}$ Department of Internal Medicine, Division of Endocrinology, Radboud University Medical Center, Nijmegen, The Netherlands. ${ }^{20}$ Department of Internal Medicine and Radboud Center for Infectious Diseases, Radboud University Medical Center, Nijmegen, The Netherlands. ${ }^{21}$ Department of Cardiology, Leiden University Medical Center, Leiden, The Netherlands. ${ }^{22}$ Netherlands Heart Institute, Utrecht, The Netherlands. ${ }^{23}$ Department of Rheumatology, Leiden University Medical Center, Leiden, The Netherlands. ${ }^{24}$ Department of Orthopaedics, Leiden University Medical Center, Leiden, The Netherlands. ${ }^{25}$ Institute for Evidence-Based Medicine in Old Age (IEMO), Leiden, The Netherlands. ${ }^{26}$ Department of Public Health and Primary Care, Leiden University Medical Center, Leiden, The Netherlands. ${ }^{27}$ BHF Glasgow Cardiovascular Research Centre, Faculty of Medicine, Glasgow, UK. ${ }^{28}$ Max Planck Institute for Biology of Ageing, Cologne, Germany. ${ }^{29}$ Institute for Community Medicine, University Medicine Greifswald, Greifswald, Germany. ${ }^{30}$ Department of Human Genetics, Leiden University Medical Center, Leiden, The Netherlands. ${ }^{31}$ Department of Internal Medicine, Division of Endocrinology, Leiden University Medical Center, Leiden, The Netherlands. ${ }^{32}$ Einthoven Laboratory for Experimental Vascular Medicine, Leiden University Medical Center, Leiden, The Netherlands.

Received: 12 May 2021 Accepted: 16 September 2021

Published online: 03 November 2021

\section{References}

1. Duntas LH. Thyroid disease and lipids. Thyroid. 2002;12(4):287-93. https:// doi.org/10.1089/10507250252949405.

2. Liu XL, He S, Zhang SF, Wang J, Sun XF, Gong CM, et al. Alteration of lipid profile in subclinical hypothyroidism: a meta-analysis. Med Sci Monit. 2014; 20:1432-41. https://doi.org/10.12659/MSM.891163.

3. Rodondi N, den Elzen WP, Bauer DC, Cappola AR, Razvi S, Walsh JP, et al. Subclinical hypothyroidism and the risk of coronary heart disease and mortality. Jama. 2010;304(12):1365-74. https://doi.org/10.1001/jama.2010.13 61.

4. Stott DJ, Rodondi N, Kearney PM, Ford I, Westendorp RGJ, Mooijaart SP, et al. Thyroid hormone therapy for older adults with subclinical hypothyroidism. N Engl J Med. 2017;376(26):2534-44. https://doi.org/10.1 056/NEJMoa1603825. 
5. Mooijaart SP, Du Puy RS, Stott DJ, Kearney PM, Rodondi N, Westendorp RGJ, et al. Association between levothyroxine treatment and thyroid-related symptoms among adults aged 80 years and older with subclinical hypothyroidism. Jama. 2019;322(20):1-11. https://doi.org/10.1001/jama.2019.17274.

6. Stott DJ, Gussekloo J, Kearney PM, Rodondi N, Westendorp RG, Mooijaart S, et al. Study protocol; Thyroid hormone Replacement for Untreated older adults with Subclinical hypothyroidism - a randomised placebo controlled Trial (TRUST). BMC Endocr Disord. 2017;17(1):6. https://doi.org/10.1186/s12 902-017-0156-8.

7. Holmes MV, Ala-Korpela M, Smith GD. Mendelian randomization in cardiometabolic disease: challenges in evaluating causality. Nat Rev Cardiol. 2017;14(10):577-90. https://doi.org/10.1038/nrcardio.2017.78.

8. Cappola AR, Desai AS, Medici M, Cooper LS, Egan D, Sopko G, et al. Thyroid and cardiovascular disease research agenda for enhancing knowledge, prevention, and treatment. Circulation. 2019;139(25):2892-909. https://doi. org/10.1161/CIRCULATIONAHA.118.036859.

9. van Vliet NA, Noordam R, van Heemst D. Thyroid hormone therapy for older adults with subclinical hypothyroidism. N Engl J Med. 2017;377(14): e20. https://doi.org/10.1056/NEJMc1709989.

10. Zhao JV, Schooling CM. Thyroid function and ischemic heart disease: a Mendelian randomization study. Sci Rep. 2017;7(1):8515. https://doi.org/10.1 038/s41598-017-07592-z

11. Larsson SC, Allara E, Mason AM, Michaelsson K, Burgess S. Thyroid function and dysfunction in relation to 16 cardiovascular diseases. Circ Genomic Precision Med. 2019;12(3):e002468. https://doi.org/10.1161/CIRCGEN.118. 002468.

12. Marouli E, Kus A, Del Greco MF, Chaker L, Peeters R, Teumer A, et al. Thyroid function affects the risk of stroke via atrial fibrillation: a Mendelian Randomization study. J Clin Endocrinol Metab. 2020;105(8):2634-41. https:// doi.org/10.1210/clinem/dgaa239.

13. Hollowell JG, Staehling NW, Flanders WD, Hannon WH, Gunter EW, Spencer CA, et al. Serum TSH, T4, and thyroid antibodies in the United States population (1988 to 1994): National Health and Nutrition Examination Survey (NHANES III). J Clin Endocrinol Metab. 2002;87(2):489-99. https://doi. org/10.1210/jcem.87.2.8182

14. McLeod DS, Cooper DS, Ladenson PW, Whiteman DC, Jordan SJ. Race/ ethnicity and the prevalence of thyrotoxicosis in young Americans. Thyroid. 2015;25(6):621-8. https://doi.org/10.1089/thy.2014.0504.

15. Chi GC, Kanter MH, Li BH, Qian L, Reading SR, Harrison TN, et al. Trends in acute myocardial infarction by race and ethnicity. J Am Heart Assoc. 2020; 9(5):e013542. https://doi.org/10.1161/JAHA.119.013542.

16. Teumer A, Chaker L, Groeneweg S, Li Y, Di Munno C, Barbieri C, et al. Genome-wide analyses identify a role for SLC17A4 and AADAT in thyroid hormone regulation. Nat Commun. 2018;9(1):4455. https://doi.org/10.1038/ s41467-018-06356-1.

17. Oresic M. Metabolomics, a novel tool for studies of nutrition, metabolism and lipid dysfunction. Nutr Metab Cardiovasc Dis. 2009;19(11):816-24. https://doi.org/10.1016/j.numecd.2009.04.018.

18. Blauw LL, Noordam R, Soidinsalo S, Blauw CA, Li-Gao R, de Mutsert R, et al. Mendelian randomization reveals unexpected effects of CETP on the lipoprotein profile. Eur J Hum Genet. 2019;27(3):422-31. https://doi.org/10.1 038/s41431-018-0301-5.

19. Janovsky C, Bittencourt MS, Goulart AC, Santos RD, Blaha MJ, Jones S, et al. Unfavorable triglyceride-rich particle profile in subclinical thyroid disease: a cross-sectional analysis of ELSA-Brasil. Endocrinology. 2021;162(2). https:// doi.org/10.1210/endocr/bqaa205.

20. Janovsky C, Generoso G, Goulart AC, Santos RD, Blaha MJ, Jones S, et al. Differences in HDL particle size in the presence of subclinical thyroid dysfunctions: the ELSA-Brasil study. Atherosclerosis. 2020;312:60-5. https:// doi.org/10.1016/j.atherosclerosis.2020.08.021.

21. Lawlor DA, Tilling K, Davey Smith G. Triangulation in aetiological epidemiology. Int J Epidemiol. 2016;45(6):1866-86. https://doi.org/10.1093/ ije/dyw314.

22. Ter Horst R, Jaeger M, Smeekens SP, Oosting M, Swertz MA, Li Y, et al. Host and environmental factors influencing individual human cytokine responses. Cell. 2016;167(4):1111-1124.e1113.

23. Riyazi N, Meulenbelt I, Kroon HM, Ronday KH, Hellio le Graverand MP, Rosendaal FR, et al. Evidence for familial aggregation of hand, hip, and spine but not knee osteoarthritis in siblings with multiple joint involvement: the GARP study. Ann Rheum Dis. 2005;64(3):438-43. https://doi.org/10.113 6/ard.2004.024661
24. Altmann-Schneider I, de Craen AJM, Slagboom PE, Westendorp RGJ, van Buchem MA, Maier AB, et al. Brain tissue volumes in familial longevity: the Leiden Longevity Study. Aging Cell. 2012;11(6):933-9. https://doi.org/1 0.1111/j.1474-9726.2012.00868.x.

25. Penninx BW, Beekman AT, Smit JH, Zitman FG, Nolen WA, Spinhoven P, et al. The Netherlands Study of Depression and Anxiety (NESDA): rationale, objectives and methods. Int J Methods Psychiatr Res. 2008;17(3):121-40. https://doi.org/10.1002/mpr.256.

26. Shepherd J, Blauw GJ, Murphy MB, Cobbe SM, Bollen EL, Buckley BM, et al. The design of a prospective study of Pravastatin in the Elderly at Risk (PROSPER). PROSPER Study Group. PROspective Study of Pravastatin in the Elderly at Risk. Am J Cardiol. 1999;84(10):1192-7. https://doi.org/10.1016/ S0002-9149(99)00533-0.

27. Ikram MA, Brusselle GGO, Murad SD, van Duijn CM, Franco OH, Goedegebure A, et al. The Rotterdam Study: 2018 update on objectives, design and main results. Eur J Epidemiol. 2017;32(9):807-50. https://doi. org/10.1007/s10654-017-0321-4.

28. Volzke H, Alte D, Schmidt CO, Radke D, Lorbeer R, Friedrich N, et al. Cohort profile: the study of health in Pomerania. Int J Epidemiol. 2011;40(2):294307. https://doi.org/10.1093/ije/dyp394.

29. Kettunen J, Demirkan A, Wurtz P, Draisma HH, Haller T, Rawal R, et al. Genome-wide study for circulating metabolites identifies 62 loci and reveals novel systemic effects of LPA. Nat Commun. 2016;7(1):11122. https://doi. org/10.1038/ncomms11122.

30. Karpe F, Vasan SK, Humphreys SM, Miller J, Cheeseman J, Dennis AL, et al. Cohort profile: the Oxford biobank. Int J Epidemiol. 2018;47(1):21-21 g.

31. de Mutsert R, den Heijer M, Rabelink TJ, Smit JW, Romijn JA, Jukema JW, et al. The Netherlands Epidemiology of Obesity (NEO) study: study design and data collection. Eur J Epidemiol. 2013;28(6):513-23. https://doi.org/10.1 007/s10654-013-9801-3.

32. Elliott $P$, Vergnaud AC, Singh D, Neasham D, Spear J, Heard A. The Airwave Health Monitoring Study of police officers and staff in Great Britain: rationale, design and methods. Environ Res. 2014;134:280-5. https://doi. org/10.1016/j.envres.2014.07.025.

33. Soininen P, Kangas AJ, Würtz P, Suna T, Ala-Korpela M. Quantitative serum nuclear magnetic resonance metabolomics in cardiovascular epidemiology and genetics. Circ Cardiovasc Genet. 2015;8(1):192-206. https://doi.org/10.11 61/CIRCGENETICS.114.000216

34. Dona AC, Jimenez B, Schafer H, Humpfer E, Spraul M, Lewis MR, et al. Precision high-throughput proton NMR spectroscopy of human urine, serum, and plasma for large-scale metabolic phenotyping. Anal Chem. 2014 86(19):9887-94. https://doi.org/10.1021/ac5025039.

35. Petersen M, Dyrby M, Toubro S, Engelsen SB, Norgaard L, Pedersen HT, et al. Quantification of lipoprotein subclasses by proton nuclear magnetic resonance-based partial least-squares regression models. Clin Chem. 2005; 51(8):1457-61. https://doi.org/10.1373/clinchem.2004.046748.

36. Karaman I, Ferreira DL, Boulange $\mathrm{CL}$, Kaluarachchi MR, Herrington D, Dona $A C$, et al. Workflow for integrated processing of multicohort untargeted (1)H NMR metabolomics data in large-scale metabolic epidemiology. J Proteome Res. 2016;15(12):4188-94. https://doi.org/10.1021/acs.jproteome.6b00125.

37. Schunkert $H$, Konig IR, Kathiresan S, Reilly MP, Assimes TL, Holm H, et al. Large-scale association analysis identifies 13 new susceptibility loci for coronary artery disease. Nat Genet. 2011;43(4):333-8. https://doi.org/10.103 8/ng.784.

38. Sudlow C, Gallacher J, Allen N, Beral V, Burton P, Danesh J, et al. UK biobank: an open access resource for identifying the causes of a wide range of complex diseases of middle and old age. PLoS Med. 2015;12(3):e1001779. https://doi.org/10.1371/journal.pmed.1001779.

39. Tabassum R, Ramo JT, Ripatti P, Koskela JT, Kurki M, Karjalainen J, et al. Genetic architecture of human plasma lipidome and its link to cardiovascular disease. Nat Commun. 2019;10(1):4329. https://doi.org/10.103 8/s41467-019-11954-8.

40. Gu Z, Gu L, Eils R, Schlesner M. Brors B: circlize Implements and enhances circular visualization in R. Bioinformatics. 2014;30(19):2811-2. https://doi. org/10.1093/bioinformatics/btu393.

41. Gu Z, Eils R, Schlesner M. Complex heatmaps reveal patterns and correlations in multidimensional genomic data. Bioinformatics. 2016;32(18): 2847-9. https://doi.org/10.1093/bioinformatics/btw313.

42. Lawlor DA. Commentary: Two-sample Mendelian randomization: opportunities and challenges. Int J Epidemiol. 2016;45(3):908-15. https://doi. org/10.1093/ije/dyw127. 
43. Bowden J, Holmes MV. Meta-analysis and Mendelian randomization: a review. Res Synth Methods. 2019;10(4):486-96. https://doi.org/10.1002/ jrsm.1346.

44. Verbanck M, Chen C-Y, Neale B, Do R. Detection of widespread horizontal pleiotropy in causal relationships inferred from Mendelian randomization between complex traits and diseases. Nat Genet. 2018;50(5):693-8. https:// doi.org/10.1038/s41588-018-0099-7.

45. R Core Team. R: a language and environment for statistical computing. In., 3.6.1 edn. Vienna: R Foundation for Statistical Computing; 2019.

46. Hemani G, Zheng J, Elsworth B, Wade KH, Haberland V, Baird D, et al. The MR-Base platform supports systematic causal inference across the human phenome. Elife. 2018;7. https://doi.org/10.7554/eLife.34408.

47. Viechtbauer W. Conducting meta-analyses in $\mathrm{R}$ with the metafor package. J Stat Softw. 2010;36(3):48. https://doi.org/10.18637/jss.v036.i03.

48. Wickham H. ggplot2: elegant graphics for data analysis. New York: SpringerVerlag; 2009.

49. Scheinin I, Kalimeri M, Jagerroos V, Parkkinen J, Tikkanen E, Würtz P, et al. ggforestplot: Forestplots of measures of effects and their confidence intervals; 2020

50. Pietzner M, Kacprowski T, Friedrich N. Empowering thyroid hormone research in human subjects using OMICs technologies. J Endocrinol. 2018 238(1):R13-r29. https://doi.org/10.1530/JOE-18-0117.

51. Klieverik LP, Coomans CP, Endert E, Sauerwein HP, Havekes LM, Voshol PJ, et al. Thyroid hormone effects on whole-body energy homeostasis and tissue-specific fatty acid uptake in vivo. Endocrinology. 2009;150(12):563948. https://doi.org/10.1210/en.2009-0297.

52. Sinha RA, Singh BK, Yen PM. Direct effects of thyroid hormones on hepatic lipid metabolism. Nat Rev Endocrinol. 2018;14(5):259-69. https://doi.org/10.1 038/nrendo.2018.10

53. Duntas $L H$, Brenta G. A renewed focus on the association between thyroid hormones and lipid metabolism. Front Endocrinol (Lausanne). 2018;9:511. https://doi.org/10.3389/fendo.2018.00511.

54. Holmes MV, Millwood IY, Kartsonaki C, Hill MR, Bennett DA, Boxall R, et al. Lipids, lipoproteins, and metabolites and risk of myocardial infarction and stroke. J Am Coll Cardiol. 2018;71(6):620-32. https://doi.org/10.1016/j.jacc.2 017.12.006.

55. Razvi S, Jabbar A, Pingitore A, Danzi S, Biondi B, Klein I, et al. Thyroid hormones and cardiovascular function and diseases. J Am Coll Cardiol. 2018;71(16):1781-96. https://doi.org/10.1016/j.jacc.2018.02.045.

56. Kuś A, Chaker L, Teumer A, Peeters RP, Medici M. The genetic basis of thyroid function: novel findings and new approaches. J Clin Endocrinol Metab. 2020;105(6). https://doi.org/10.1210/dlinem/dgz225.

57. Ellervik C, Roselli C, Christophersen IE, Alonso A, Pietzner M, Sitlani CM, et al. Assessment of the relationship between genetic determinants of thyroid function and atrial fibrillation: a Mendelian randomization study. JAMA Cardiol. 2019:4(2):144-52. https://doi.org/10.1001/jamacardio.2018.4635.

58. Hemani G, Bowden J, Davey Smith G. Evaluating the potential role of pleiotropy in Mendelian randomization studies. Hum Mol Genet. 2018 27(R2):R195-r208. https://doi.org/10.1093/hmg/ddy163.

59. Smit RAJ, Trompet S, Dekkers OM, Jukema JW, le Cessie S. Survival bias in Mendelian randomization studies: a threat to causal inference. Epidemiology. 2019;30(6):813-6. https://doi.org/10.1097/EDE.0000000000001 072 .

60. Haworth S, Mitchell R, Corbin L, Wade KH, Dudding T, Budu-Aggrey A, et al. Apparent latent structure within the UK Biobank sample has implications for epidemiological analysis. Nat Commun. 2019;10(1):333. https://doi.org/10.1 038/541467-018-08219-1.

61. Burgess S, Davies NM, Thompson SG. Bias due to participant overlap in twosample Mendelian randomization. Genet Epidemiol. 2016:40(7):597-608. https://doi.org/10.1002/gepi.21998.

\section{Publisher's Note}

Springer Nature remains neutral with regard to jurisdictional claims in published maps and institutional affiliations.

Ready to submit your research? Choose BMC and benefit from:

- fast, convenient online submission

- thorough peer review by experienced researchers in your field

- rapid publication on acceptance

- support for research data, including large and complex data types

- gold Open Access which fosters wider collaboration and increased citations

- maximum visibility for your research: over $100 \mathrm{M}$ website views per year

At $\mathrm{BMC}$, research is always in progress.

Learn more biomedcentral.com/submissions 